\title{
The Role of E-Tourism Mediation in the Relationship between Climate Change and the amount of Income from Tourism Industry in Iran
}

\author{
Seyyed Mohammad Ghaem Zabihi \\ MSC student of Economics, Ferdowsi University of Mashhad, Mashhad, Iran \\ Smq.Zabihi@mail.um.ac.ir \\ Khashayar Safarzaei \\ BSc. in Economics, University of Sistan and Baluchestan, Zahedan, Iran \\ Tankianserj95@yahoo.com
}

\begin{abstract}
In the recent century, the tourism industry and within it the tourism economy are one of the most important and fundamental sectors of engaged business. E-tourism can be used as a dynamic tool in up to date areas of informative information and tourism marketing will be considered as a suitable field for the tourism industry. The aim of this study was to investigate the relationship between climate change and the amount of revenues from the tourism industry relying on a tool called e-tourism, and informing and providing services through this way so that Iran can achieve a greater share of export of a singleproduct oil economy combined with economic growth and sustainable development goals. The method of this research is descriptiveanalytical.
\end{abstract}

Keywords: E-tourism, Climate, Climate Change, Tourism Industry

JEL Classification: D83, Q54, L83, Z32 


\section{Introduction}

In the new age of tourism industry as one of the largest industries and the industry's most important subsidiaries and trade in the world, it is considered as the industry with high employment income. The industry has created demand for many goods and services in global markets and has a great impact on other sectors including airlines, tour operators, travel agencies, and the prosperity of the residences and many other economic aspects. On the other hand, the industry has a lot of dependence on the climate of a region and many tourism destinations have been a thriving tourist resource in certain seasons of the year. In this regard, it can be acknowledged that both tourists and tourism industry are required to have enough information regarding climate. Thus, the availability of suitable climatic information for any tourist before, after and during travel is necessary.

Before traveling, the tourist can achieve this information through the media and the meteorology organizations, while during the trip the daily weather information about the short-term planning of traveler is obtained and finally after the trip this information can be useful for setting subsequent trips to that destination. On the other hand, climate can be used as an effective factor in tourism planning and decision making (Dogru et al., 2019).

since, the health benefits of climatology have encouraged the scope of researchers to study the various aspects of tourism climate in the field of tourism planning and leisure processes. The research fields in this regard have been increased from decision-making activities to the construction and development of tourism facilities or the determination of the entertainment season, technical needs and necessary facilities, determining the appropriate areas for tourism with the aim of helping tourists in choosing the right areas for travel.

But today, as one of the most important industries in the development of various nations ' cultures and associations, the tourism plays a great role in the establishment of peace and tranquility in the world and the tourism industry is considered as one of the profitable, yet healthy and clean industries for the economy of every country. In the world of development, information and communication technology has a profound impact on tourism and travel, and this is still changing its structure and processes. The tourism industry has to be more flexible, more efficient than the past in responding to the demands of customers (Buhalis, 2003: 35).

In general, tourism is a combination of activities, services and industries that provide a joyfull travel. Tourism has some important types: cultural tourism, rural tourism, urban tourism, religious tourism, health tourism, commercial tourism and e-tourism. In the topics ahead we will work more with the last type of it; electronic tourism. With the enhanced communities and innovation in the information sector, tourism is transformed and variable. In this type of tourism, the individual tourists can easily learn all geographical information, products, and any other necessary informations (Amelung and Alaro, 2009).

Iran is also a part of the tourism world and is considered as a major infrastructure in tourism, and of course, everyone believe that IRAN can have a significant contribution in tourism. The aim of the present study is to explain that the tourism industry can act as a income generating sector in the Iranian economy appended from relying on oil revenues and single-product economy (Bigano et al., 2006). In fact, by examining the effective factors on a branch of the tourism industry, electronic tourism and providing solutions in this format, it is shown that this section can help the country's economy.

Considering the importance of electronic tourism in the tourism industry, in the relevant literature, the research, which directly examines the mediating role of electronic tourism in the relationship between climate change and the amount of revenues from the tourism industry in Iran, was achieved. Also, considering the importance and necessity of climate change on tourism, the present study was designed to investigate the mediating role of e-tourism in the relationship between climate change and the amount of income from tourism industry in Iran. Previous studies have been mentioned on the subject of further research.

Sokhanvar (2019), has studied that, "does direct foreign investment on tourism accelerate economic growth in Europe?". The result is achieved in its research shown that foreign direct 
investment has a negative impact on economic growth of five European countries, and surprisingly the tourism industry does not stimulate any of our typical countries. Dogru et al. (2019), in an article titled "Climate Change: the vulnerability and flexibility of tourism and the entire economy" achieve this conclusion that the tourism industry is more vulnerable to climate change than the whole economy. The power of these influences varies in countries with different income levels: countries with minimal income are more vulnerable and flexible, and those with highest income are the least vulnerable and flexible. Liu et al. (2019), "The impact of climate-related changes to the time of coloring of the leaves of autumn trees on Japanese tourism" in this study, the delay in the staining of autumn foliage and leaf loss periods, will increase the rate of maple tourism in December, 3.64 and 3.02\%, respectively. The effect on the autumn leaves of the view of tourism foliage also has a one-year delay, and the foliage of maple trees in different cities has a significant impact on tourism volume. In general, changes in the time changed maple leaf color for the Japanese tourism industry has been beneficial. Christopher Craig (2018), in an article titled "Temporal and Spatial analysis of climate change, climate events and Tourism Jobs" explores how to change precipitation and temperature across the United States in several outdoor tourism locations in six unique climatic areas from 1990 to 2015 compared to the long-term averages of the year 1901. In this study, it is the first time to use a correlation-level theory to explore a small and longitudinal view of the geographical and time of climate change and extreme events in business results. The methods used, including the prediction of time series, provide a blue print for high-risk jobs in order to examine the impact of climatic factors and climate conditions, regardless of location. Hewer et al. (2018), in an article titled "Thirty Years of evaluating the impacts of climate change in outdoor recreation and Tourism in Canada" examines the 30 articles of the university magazine (1986 - 2016), which evaluates the impact of climate change in recreation and outdoor tourism in Canada. In general, it is anticipated that climate change increases the risks of cold weather activities in Canada, while it may increase opportunities for activities in hot air. A series of identified knowledge gaps and recommendations for future research are presented in this area. The emphasis on overcoming constraints related to reliance on outdated weather science, climatic models and scenario changes. Addressing uneven geographical distribution of existing assessments and filling gaps in the areas that are currently less representative. As well as examining water sensitivity and possible impacts of climate change for outdoor recreation and tourism activities that have not yet been evaluated.

\section{Literature review}

Tourism has combined two sections "TOUR" means travel, patrol and "ISM", which refers to the school or thought of philosophical, religious, political, literary, etc. It means that the intellectual base of the tourism is a tourist (Oxford vocabulary, 1989:1356). The historic journey of this word from Greece to Spanish and French has finally been in English.

In general, the tourist is a person who travels for a period of time, at least one night and less than a year (12months), to a country other than their native homeland or residence, and his goal is not to work and earn money (International Tourism Organization, 1999). Tourism is one of the important factors of wealth and employment in the world and as the economic engine of growth and development of developed and developing countries in the world. During the recent decades, the tourism industry in the world has resulted in the development of significantly and economic and social changes. One of the most effective ways and debts in the country is to achieve economic growth and more specifically sustainable development, expanding the level of tourism activities.

\section{2-1. E-Tourism}

E-Tourism (ET) is a technology of integration of electronic business methods and information technology in the ways and tools of procurement, delivery and support of tourism services for the services required by tourists with higher quality and less cost . In fact, Etis electronic Presentation of 
all services in the past that tourists used traditionally in addition to services that are available through information technology. From another perspective in general, all components of business such as ecommerce, research and development of electronics, content production and provision of services in the form of electronics in cyberspace or communication are included. Also, e-tourism refers to a collection of Web sites (public and private) and specific standards and protocols that produce and redistribute the information in various formats such as text, image, catalog and brochures, advertising and even services such as hotel reservations and e-shopping (Scott et al., 2008).

For the importance of this issue, the slogan of the International Tourism Day celebrated on September 27, 2018 was "digital tourism and transformation." This slogan and the emphasis on the importance of tourism has updated and digitized in use.

The discussion we want to do here is that e-tourism does not mean circulation or travel electronically. Although virtual trips can also be a subset of the activities in the sector. The concept of e-tourism includes all components of business such as: e-commerce, e-marketing, e-Financial, e-accounting, electronic human resources management, electronic research and development and electronic production as well as electronic strategies including electronic planning and management for all sectors of the industry.

If, for example, an Iranian website (that sales only Iranian goods), here, people who access this site from abroad can be considered as a virtual and electronic tourist, which it is in accordance with the definitions of the tourist, for the purpose of non-job search of this virtual space and the Iranian web site are visited. So it can be a newer definition and a more innovative aspect of electronic tourism.

\section{2-2. Climate Change}

Usually, at any specified location, during the year, weather can change. In terms of abundance. This air is calculated in every region, water and region. So the climate is: the dominant air in a longterm place (Alikhani and Kaviani, 1384, 18). The word of the climate, which means that in Persian, the country, a certain territory that is the same weather situation, is from the Greek word. The collection of air statistics that interfere with the description of a place or region is called climate (Viner and Amelong, 2003).

Considering the importance of the tourism industry, the necessity of recognizing favorable and unfavorable climatic conditions for domestic and foreign tourists is essential, the general characteristics of the destination climate and daily, monthly and seasonal changes, temperature, precipitation, humidity, radiation, wind and other climate elements for tourism destination are important information for the applicant to be able to fit it into their planning in terms of departure time, type of clothing and equipment required (Hein et al., 2009). Climate is among the most effective structural factors of planet Earth and undoubtedly, nature, human and all manifestations of life are influenced by wide range of climatic conditions. Tourists usually search for a favorable climate , where the person has no sense of dissatisfaction and lack of thermal and climatic comfort and this factor plays an important role in deciding on the tourism destination (Matzarakis, 2004, 26).

\section{2-3. Climate impacts on tourist and tourism industry}

In a general view, the climate can be effective in the tourism industry, according to the parameters in each of them as a graph and a framework (Fig.1). The tourism climate consists of three main components that include: the thermal effects (air temperature, humidity, wind, sunlight intensity, and long wavelength of the solar radiation are effective in warmth and surface coldness), the physical effects (dust and annoying loads, continuous precipitation and flood), unagreeable moisture and improper and inappropriate air, and aesthetic effects (favorable sun radiation, beautiful clouds and the number of sunny hours appropriate). These factors affect the behaviors and encounters of tourists in the tourism industry and eclipse demand forecasts, tourism facilities planning, travel timings (for 
travelers and towprtours) and the application of weather attractiveness in tourism marketing destination.

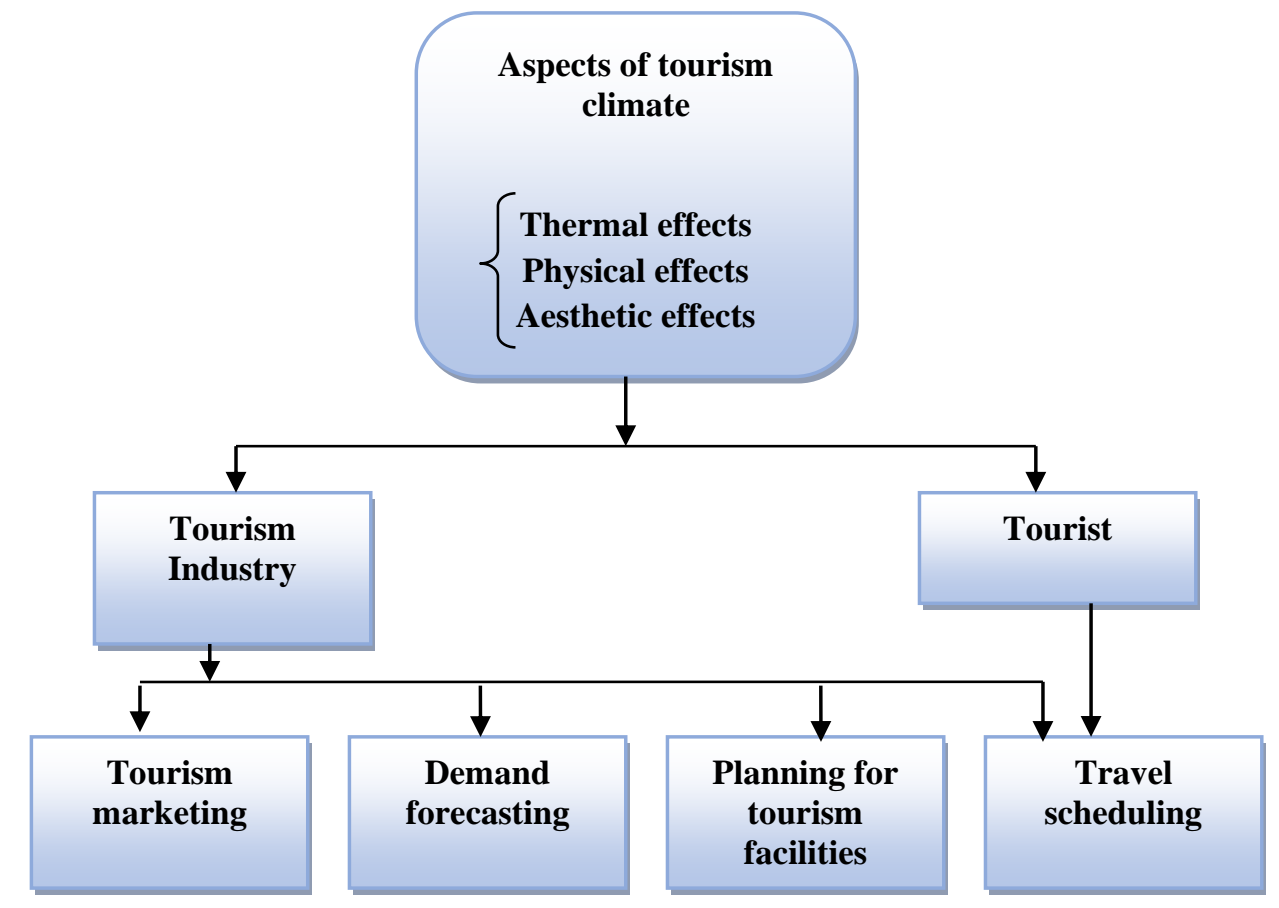

Resource: Hein et al. 2009 And Editors ' edits

Fig. 1. Conceptual Framework for Tourism studies

\section{2-4. Application of climatic indicators of tourism}

Employing a standard method to evaluate tourism climate, evaluating the role of meteorological forecasting and the impact of long-term climatic forecasts on selected tourists, the effect of atmospheric phenomena and risk phenomena in tourism industry, including the studied cases. In this regard, the International Association of Climate, Tourism and Recreation Committee was formed during the 15th Congress in 1999 in Sydney, Australia (Feritas, 2003). The purpose of this association is to expand research in the field of tourism climate. The first official meeting of the Committee was on October 5 to 10, 2001 in the Hall of Dicky, Greece.

The objectives of this meeting were to bring together experts in the field of climate and tourism to review the current situation of tourism climatology and to determine the areas and prioritize future works in line with the objectives of the Association. Workshops and meetings showed the fact that although the climate has been diagnosed as an important necessity in many aspects of tourism, it has not been given enough attentions in the studies. Also, the economic impacts and climate significance have been discussed in predicting tourism trade. Madison (2001), Lise and Tol (2002), Hamilton (2003) and Bigano et al. (2006) In their statistical analysis, the impact of climatic factors such as temperature on tourism demand have proved. Viner and Amlong (2003) has also been more widely considered in their studies on the interactions of climate and tourism and environmental changes. Although the relationship between climate and tourism demand and the satisfaction of tourists seems obvious, the logical relationship between these two has been complicated (Freitas, 2003). Hence, introduction and application of tourism climate indices are among the most effective methods in recognizing the climate and tourism industry. In this study, the role of climate in tourism is investigated and its economic benefits have been considered in the region. 


\section{2-5. Surveying the role of electronic tourism on climate change and revenues from tourism industry}

As the superior content and definitions of our tourism industry and electronic tourism in Iran, we need to follow this important step to strengthen the powerful and robust tools such as e-tourism, so that we can adopt solutions in the field of informing and attracting tourists (both domestic and foreign). Due to the fact that Iran has a four-season climate, it is very specific and strategic, it can be important to the seats, with the establishment of a series of websites in each chapter from countries that are susceptible to tourism and travel, and provide comprehensive awareness and information from the beginning of the journey to cover all the required items, so that travel applicants are able to easily Find their favorite destinations and tourist attractions and predicament a case based on their interests and tastes. For example, tourist is willing to see the steps to the operation of saffron, it can be seen as the exact date and season of this product and put its schedule in autumn in the area under saffron cultivation and witnessed its different stages. that is why we can do this with a deep and profound wisdom and bring tourists to the lost paradise of Iran, and from the same route to earn income from the tourism industry channel, and continue to take it with the rich revenues earned in our growth and development.

\section{Discussion and Results}

The industrial tourism industry is dependent on the climate and the climate itself plays a significant role as a natural attraction for tourism and regional tourism prosperity. But it has always been considered as a trivial affair and has not given enough attention to its impact on tourism planning. In this research, the author has originally attempted to reveal the dimensions and effects of tourism, followed by a tool called electronic tourism, to evolve and the maturity of the tourism industry, which is a important in the Iranian economy. That is, as it was suggested can be done by constructing a series of research teams of the analysis process, I would consider this important that all regions and provinces of Iran will be determined to attract more tourists. A number of cases such as sunny days, average temperature throughout the day and other important and prominent parameters in these areas are evaluated and as a result of this research, we have a configuration map for the country. In this format, like our neighbor country, Turkey has formed a central Web site for the resonance services ranging from reservation of flights, rooms, cars and other items that exist. Sufficient attention and investment in such cases are highly efficient, because Iran is a very rich country and is rich in tourist resources, but for many reason not able to use these resources. such as the utmost maximum use, we studied in this research, if we can use electronic tourism tools, we will be able to earn very high revenues in the field of tourism industry from four seasons and beautiful Iranian country. It is suggested that this climate change is considered separately for more tourist provinces in terms of climate change.

\section{References}

1) Amelung, Bas and M, Alvaro. (2009). Impacts of climate change in tourism in Europe. university Maastrich, 43.

2) Buhalis, D. (2003). E-Tourism:Information Technology For Strategic Tourism Management Printce hall uk. pp: 35 .

3) Bigano, A., Hamilton, J. M. and Tol, R. S. J. (2006). Impact of climate on tourism demand. Climate Change: 76: 389-406. 
4) Christopher A.Craig \& SongFeng. (2018). A temporal and spatial analysis of climate change, weather events, and tourism businesses. Tourism Management, Volume 67, August 2018, Pages 351-361.

https://doi.org/10.1016/j.tourman.2018.02.013.

5) Freitas, C. R. de. (2003). Tourism climatology: evaluating environmental information for decision making and business planning in the recreation and tourism sector. Int. J. Biometeorol, 48: 45-54.

6) Hamilton, J. M. (2003). Climate and the destination choice of German tourists. Working Paper FNU-15 (revised), Research Unit Sustainability and Global Change, Centre for Marine and Climate Research, University of Hamburg.

7) Hein, L., Metzger, M. J., \& Moreno, A. (2009). Potential impacts of climate change on tourism; a case study for Spain. Current Opinion in Environmental Sustainability, 1(2), 170-178

8) Jun Liu ,Han Cheng, Di Jiang, Li Huangm. (2018). Impact of climate-related changes to the timing of autumn foliage colouration on tourism in Japan. Tourism Management, Volume 70, February 2019, Pages 262-272 .https://doi.org/10.1016/j.tourman.2018.08.021.

9) Matzarakis. A. (2004). Assessing climate for tourism purpose: Existing methods and climate. Tourism and recreation tools for the termal complex, In Proceeding of the First International Workshop on Climate, Tourism and Recreation.

10) Maddison, D. (2001). In search of warmer climates? The impact of climate change on flows of British tourists. Climatic Change, 49 (1/2), 193-208.

11) Micah J.Hewer\& William A.Gough. (2018). Thirty years of assessing the impacts of climate change on outdoor recreation and tourism in Canada. Tourism Management Perspectives, Volume 26, April 2018, Pages 179-192. https://doi.org/10.1016/j.tmp.2017.07.003.

12) Lise, W. and Tol, R. S.J. (2002). Impact of climate on tourist demand. Climatic Change, 55 (4), 429-49.

13) Scott, D., Dawson, J., \& Jones, B. (2008). Climate change vulnerability of the US Northeast winter recreation-tourism sector. Mitigation and Adaptation Strategies for Global Change, 13(5-6), 577-596.

14) Sokhanvar, Amin. (2019). Does foreign direct investment accelerate tourism and economic growth within Europe. Tourism Management Perspectives, Volume29, PP 86-96.

15) TarikDogru ${ }^{a}$ Elizabeth A.Marchio' UmitBulut CourtneySuess'. (2018). Climate change: Vulnerability and resilience of tourism and the entire economy. Tourism Management, Volume 72, Pages 292-305. https://doi.org/10.1016/j.tourman.2018.12.010.

16) Viner, D. and Amelung, B. (2003). Climate change, the environment and tourism: The interactions. Proceedings of the Esf. 